\title{
Determinação do $17 \beta$-estradiol nas formas farmacêuticas gel e adesivo transdérmico
}

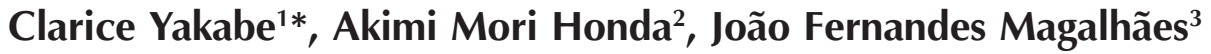 \\ ${ }^{1}$ Hospital do Servidor Público Estadual Francisco Morato de Oliveira/Serviço de Assistência Farmacêutica, ${ }^{2}$ Curso de \\ Farmácia, Faculdade Integrada de São Paulo/Gerente de P\&D da Biolab Sanus Farmacêutica Ltda, ${ }^{3}$ Departamento \\ de Farmácia, Faculdade de Ciências Farmacêuticas, Universidade de São Paulo
}

*Correspondência:

C. Yakabe

Av. Ibirapuera, 981

04029-000, Vila Clementino,

São Paulo, SP

E-mail: yakabeclarice@ig.com.br
Desenvolveu-se um método espectrofotométrico na região do visivel para doseamento de $17 \beta$-estradiol em gel e adesivo transdérmico, utilizando-se sulfanilamida diazotada como reagente. $O$ azo-composto formado apresentou absorção máxima em $480 \mathrm{~nm}$, sendo a reação linear na faixa de concentração de 10,0 a 28,0 $\mu \mathrm{g} / \mathrm{mL}$. Quando aplicado a amostras comerciais mostrou-se preciso e exato.

\section{INTRODUÇÃO}

O $17 \beta$-estradiol é um dos estrogênios usados na terapia de reposição hormonal (TRH), sendo o tratamento dos sintomas da menopausa, uma de suas indicações, devendo ser utilizado na menor dose eficaz, por um período o mais curto possível (Stephenson, 2003; Nelson, 2004).

Apesar do primeiro relatório científico sobre o uso de preparação comercial de estrogênio datar de 1935, o seu uso foi difundido nas décadas de 1950 e 1960 e, desde então, tem suscitado inúmeros debates quanto à sua segurança e eficácia (Hupert, 1987).

Atualmente, o estrogênio está disponível no mercado brasileiro nas mais diversas formas farmacêuticas (DEF2003/04), porém há carência de métodos para sua análise.

A Farmacopéia Americana preconiza o uso da cromatografia líquida de alta eficiência (CLAE) para análise da matéria-prima, creme vaginal e comprimidos, enquanto que para suspensão injetável e "pellets" continua sendo proposto o método colorimétrico com reagente fenolferro (USP, 2003). A associação dos químicos analíticos dos Estados Unidos (AOAC) também adota um método colorimétrico com reagente de ferro de Kober (fenol-ferro) para doseamento da matéria-prima (AOAC, 2000).

É inegável a eficiência dos métodos cromatográficos no controle de qualidade de medicamentos. Porém, o seu custo é alto e nem sempre facilmente aplicável às diversas formas farmacêuticas.

Na literatura estão descritos alguns métodos alternativos como o uso da voltametria (Salci, Biryol, 2002), cromatografia em camada delgada de alta eficiência (Kotiyan, Vavia, 2000), espectrofotometria derivada de segunda ordem (Toral, Soro, Richter, 2002), entre outros.

Portanto, o objetivo deste trabalho foi desenvolver e padronizar um método colorimétrico para quantificação do $17 \beta$-estradiol em gel e adesivo transdérmico. Este método, diferente dos métodos colorimétricos oficiais, é baseado na diazotação da sulfanilamida com nitrito de sódio, seguida da reação de acoplamento com $17 \beta$-estradiol. 


\section{MATERIAL E MÉTODOS}

\section{Equipamentos, reagentes e soluções}

\section{Equipamentos}

Espectrofotômetro UV/VIS Shimadzu, modelo UV1601, com impressora Epson, modelo LQ-570+; balança analítica, Ohaus, modelo AS200; banho de ultra-som, Thornton, modelo T14

\section{Reagentes e soluções}

$17 \beta$-Estradiol, padrão USP, lote KIB007 (identificação); $17 \beta$-estradiol, substância química de referência, Heartwell, lote ESMO/002, teor de 100,41\%; etanol absoluto p.a., Merck; ácido clorídrico fumegante a 37\%, Merck; carbonato de sódio anidro p.a.-ACS, Synth; cloreto de sódio PA, Quimesp; nitrito de sódio p.a.-ACS, Merck; sulfanilamida pura, Sigma; solução de carbonato de sódio $10 \%(\mathrm{p} / \mathrm{v})$; solução de cloreto de sódio $10 \%(\mathrm{p} / \mathrm{v})$; solução de nitrito de sódio $0,5 \%(\mathrm{p} / \mathrm{v})$; solução de sulfanilamida $0,1 \%$ (p/v) em solução de ácido clorídrico $0,1 \mathrm{M}$.

\section{Amostras}

Amostra comercial de gel, contendo $0,06 \mathrm{~g} \%$ de $17 \beta$ Estradiol; amostra comercial de adesivo transdérmico, contendo 3,20 mg de 17 $\beta$-estradiol; amostra simulada de gel, contendo $0,06 \mathrm{~g} \%$ de $17 \beta$-estradiol; amostra-placebo de gel.

\section{MÉTODOS}

\section{Preparação da solução-padrão}

A solução-padrão de $17 \beta$-estradiol foi preparada a partir de $100 \mathrm{mg}$ de $17 \beta$-estradiol, substância química de referência, em balão volumétrico de capacidade de $100 \mathrm{~mL}$, com adição de $80 \mathrm{~mL}$ de etanol absoluto. Colocou-se no banho de ultra-som por cinco minutos e o volume foi completado com o mesmo solvente. Procedeu-se à diluição de $20 \%$ (v/v), em etanol absoluto. A concentração final desta solução foi de $200 \mathrm{mg} / \mathrm{mL}$. A partir desta solução foram preparadas soluções para a obtenção do espectro de absorção $(8,0,16,0,24,0$ e $32,0 \mathrm{mg} / \mathrm{mL}$ de $17 \beta$-estradiol), curva de Ringbom e curva analítica.

\section{Preparação da solução-padrão para gel}

Foi preparada uma solução alcoólica de $17 \beta$-estradiol $60 \mathrm{mg} / 100 \mathrm{~mL}$. Vinte mililitros desta solução foram transferidos para um balão volumétrico de $100 \mathrm{~mL}$, contendo $20 \mathrm{~g}$ de placebo desestabilizado com $5 \mathrm{~mL}$ de cloreto de sódio $10 \%$. Após cinco minutos no banho de ultra-som, a suspensão foi filtrada em papel de filtro quantitativo, faixa branca.
Preparação da solução-padrão para adesivo transdérmico

Foi preparada uma solução alcoólica contendo $64 \mathrm{mg} / 100 \mathrm{~mL}$ de $17 \beta$-estradiol. A partir desta solução, foi feita diluição adequada para obtenção de solução contendo $128 \mathrm{mg} / \mathrm{mL}$ de $17 \beta$-estradiol.

Preparação das soluções de amostras comerciais e simuladas e placebos de gel

Tomadas de ensaio equivalentes a $12 \mathrm{mg}$ de $17 \beta$ estradiol da amostra de gel comercial, simulada e placebo foram transferidas para béqueres de $100 \mathrm{~mL}$. Adicionaramse $5 \mathrm{~mL}$ da solução de cloreto de sódio $10 \%$ e após homogeneização, foram transferidos quantitativamente para balão volumétrico de $100 \mathrm{~mL}$, com auxílio de etanol absoluto. Após cinco minutos no banho de ultra-som, os volumes foram completados com o mesmo solvente. As soluções foram filtradas através de papel de filtro quantitativo, faixa branca.

\section{Preparação da solução da amostra comercial de adesivo transdérmico}

Cada adesivo foi colocado em um béquer com $15 \mathrm{~mL}$ de etanol absoluto. Após 30 minutos no banho de ultra-som, foi transferido para um balão volumétrico de $25 \mathrm{~mL}$ e completou-se com o mesmo solvente, obtendo-se solução contendo $128 \mathrm{mg} / \mathrm{mL}$ de $17 \beta$-estradiol.

\section{Reação colorimétrica}

Em banho de gelo, misturou-se a solução de sulfanilamida $0,1 \%$ com nitrito de sódio $0,5 \%$ e incubouse por cerca de 2 minutos e, em seguida, acrescentou-se o filtrado. Agitou-se, deixando-se descansar por mais um minuto. Por fim, adicionou-se a solução de carbonato de sódio a $10 \%$ e completou-se com água destilada para $50 \mathrm{~mL}$, como indica a Tabela I.

\section{Padronização do método}

\section{Espectro de absorção}

Com as soluções-padrão contendo 8,0, 16,0, 24,0 e $32,0 \mathrm{mg} / \mathrm{mL}$ de $17 \beta$-estradiol, determinou-se o espectro de absorção do azo-composto, no intervalo de 400 a 600 nm, usando placebo como branco e cubetas de $1 \mathrm{~cm}$ de espessura.

\section{Construção da curva de Ringbom}

Preparou-se uma solução de $17 \beta$-estradiol, $200 \mathrm{mg} / \mathrm{mL}$, em etanol absoluto. A curva de Ringbom foi construída com os valores de transmitâncias do azo-compostos formados pela reação entre $17 \beta$-estradiol, nas con- 
TABELA I - Preparação das soluções para reação colorimétrica das amostras comerciais e simuladas

\begin{tabular}{lccccc}
\hline Balão & $\begin{array}{c}\text { Sulfanilamida } \\
0,1 \%(\mathrm{~mL})\end{array}$ & $\begin{array}{c}\text { Nitrito de } \\
\text { sódio } 0,5 \%(\mathrm{~mL})\end{array}$ & Filtrado $(\mathrm{mL})$ & $\begin{array}{c}\text { Carbonato de } \\
\text { sódio } 10 \%(\mathrm{~mL})\end{array}$ & $\begin{array}{c}\text { Água destilada } \\
\text { q.s.p para }(\mathrm{mL})\end{array}$ \\
\hline Padrão & 10 & 10 & 10 & 5 & 50 \\
Amostra & 10 & 10 & 10 & 5 & 50 \\
Branco (Placebo) & 10 & 10 & 10 & 5 & 50 \\
\hline
\end{tabular}

Concentração final da amostra adesivo $(25,6 \mathrm{mg} / \mathrm{mL})$, gel $(24,0 \mathrm{mg} / \mathrm{mL})$

centrações de 2,0 a $50,0 \mathrm{mg} / \mathrm{mL}$, e a sulfanilamida diazotada. As leituras das transmitâncias foram realizadas em $480 \mathrm{~nm}$.

\section{Construção da curva analítica}

A relação entre as absorvâncias do azo-compostos, em $480 \mathrm{~nm}$, e as correspondentes concentrações do $17 \beta$ estradiol, na faixa de 10,0 a $28,0 \mathrm{mg} / \mathrm{mL}$, foi utilizada para construção da curva analítica e para calcular o coeficiente de correlação e a equação da curva analítica, empregando o programa Excel 2000.

\section{Aplicação do método}

Para avaliar a eficiência do método, efetuou-se a sua padronização, empregando-se para análises, amostras comerciais, amostras simuladas e respectivos placebos.

De cada amostra dos medicamentos (comerciais e simulados) foram efetuados 10 ensaios para o gel e 6 para adesivo conforme procedimento descrito em preparo das soluções-amostra.

Os dados analíticos foram utilizados para determinação da precisão, pelo cálculo do desvio-padrão relativo intradia.

$\mathrm{O}$ teste de linearidade foi realizado adicionando-se ao placebo da formulação simulada cinco diferentes concentrações de $17 \beta$-estradiol, substância química de referência $(50,75,100 \%)$, em relação ao teor declarado, correspondendo a $12,0,18,0$ e $24,0 \mathrm{mg} / \mathrm{mL}$, respectivamente, tendose realizado o teste em triplicata, repetindo-se por três dias (ICH-Q2B, 1996).

O teste de recuperação do padrão, realizado com adição de quantidades conhecidas de padrão, em três concentrações diferentes, às porções equivalentes à metade da concentração do princípio ativo da amostra, previamente analisada, foi empregado para avaliar a exatidão do método (USP, 2003; ICH-Q2B, 1996).

\section{RESULTADOS E DISCUSSÃO}

A eficiência dos métodos cromatográficos aplicados ao controle de qualidade de medicamentos, na atualidade, é do nosso conhecimento. Porém, nem sempre é possível fazer o seu uso, ou porque o custo é elevado, ou devido à complexidade das formulações, há necessidade de um prétratamento da própria amostra, dificultando a sua execução. À vista disso, foi desenvolvido um método espectrofotométrico, que por ser fácil e econômico, pode-se adequar a qualquer laboratório.

O método baseou-se na diazotação da sulfanilamida e posterior acoplamento com $17 \beta$-estradiol. O azo-composto formado possui uma coloração marrom-avermelhada, estável por pelo menos duas horas.

O método proposto utiliza etanol absoluto para extração da substância ativa com ajuda do ultra-som. A reação colorimétrica é realizada no filtrado alcoólico.

$\mathrm{O}$ método padronizado, aplicado às amostras de medicamentos, foi avaliado quanto à precisão e exatidão, conforme descrito na Farmacopéia (USP, 2003).

Em se tratando de método espectrofotométrico, foi inicialmente verificado o seu comportamento espectral. O espectro de absorção do produto obtido da reação de acoplamento do17 $\beta$-estradiol, nas concentrações 8,0, 16,0,

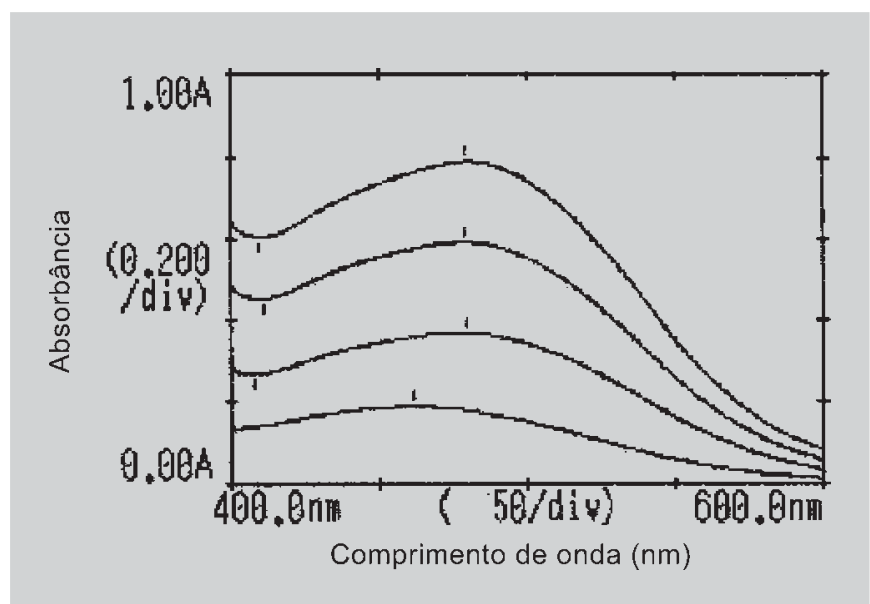

FIGURA 1 - Espectro de absorção do produto formado na reação de acoplamento da sulfanilamida diazotada e o $17 \beta$ estradiol presente nas concentrações de $8,0,16,0,24,0$ e $32,0 \mu \mathrm{g} / \mathrm{mL}$. 
24,0 e $32,0 \mathrm{mg} / \mathrm{mL}$, e a sulfanilamida diazotada mostraram curvas de absorção com boa simetria e com um máximo de absorção em 480 nm (Figura 1).

A curva de Ringbom traçada nas condições definidas está apresentada na Figura 2. A melhor faixa de concentração para ser utilizada na quantificação do $17 \beta$ estradiol ficou estabelecida entre 10,0 e $28,0 \mathrm{mg} / \mathrm{mL}$ (Figura 3).
A curva analítica obtida da relação entre valores de absorbância dos produtos coloridos da reação de acoplamento da sulfanilamida diazotada com $17 \beta$-estradiol, em diferentes concentrações, definida pela equação da reta $y=0,0273 x-0,0751$ e com um coeficiente de correlação (r) de 0,9996 está ilustrada na Figura 3.

O método espectrofotométrico de análise do $17 \beta$ estradiol, por reação de acoplamento com a sulfanilamida

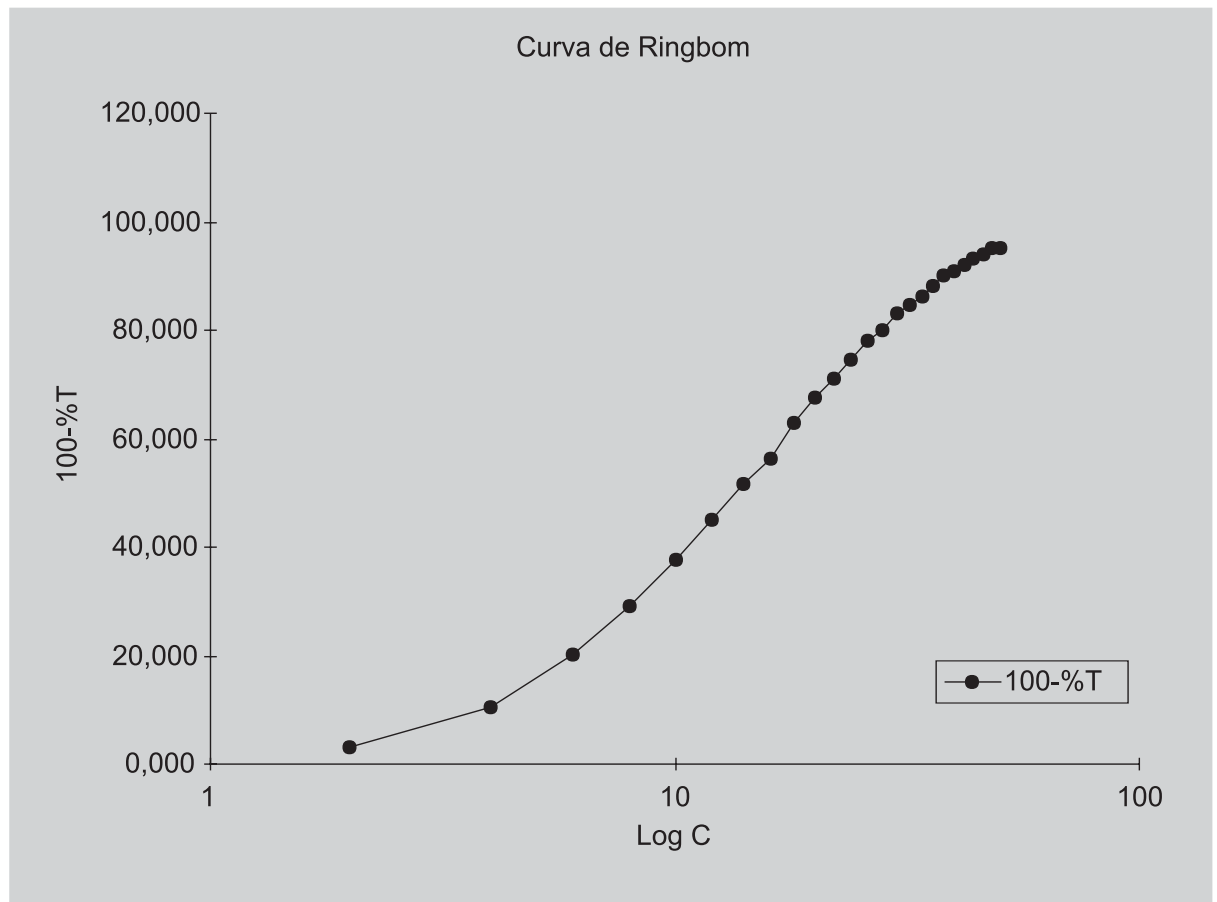

FIGURA 2 - Curva de Ringbom do produto corado resultante da reação entre 17ß-estradiol e sulfanilamida diazotada, em $480 \mathrm{~nm}$.

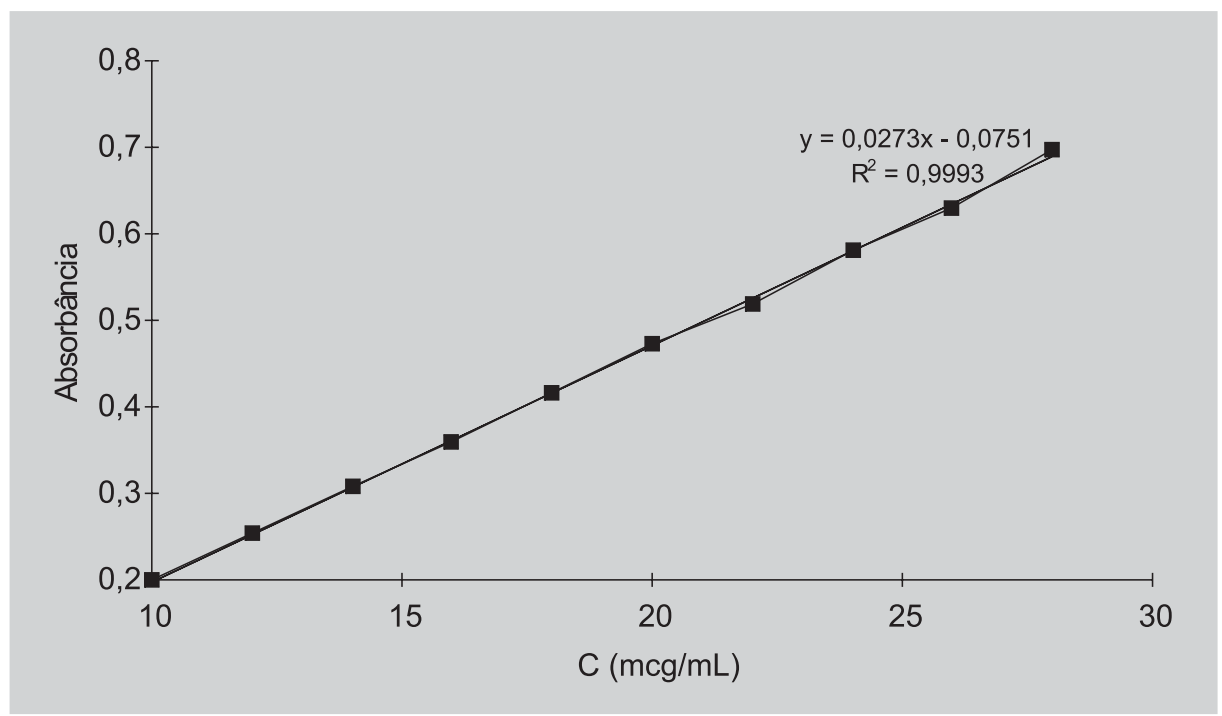

FIGURA 3 - Curva analítica obtida dos valores de absorvâncias, em $480 \mathrm{~nm}$, das soluções coradas do produto de acoplamento, resultante da reação da sulfanilamida diazotada e o $17 \beta$-estradiol nas concentrações de 10,0 a 28,0 $\mu \mathrm{g} / \mathrm{mL}$. 
diazotada, foi padronizado quanto aos parâmetros precisão intradia (repetibilidade) (Tabela II), recuperação (Tabela III) e linearidade para forma farmacêutica gel (Tabela IV e Figura 4). A precisão do método para medida do estradiol em amostras de gel simulado, gel e adesivo comerciais foi de 1,43, 0,98 e 1,60\% respectivamente, que são compatíveis com os critérios de aceitabilidade adotados pelos laboratórios de controle de qualidade de medicamentos, como também pela USP, 2003. Os resultados do teste de recupe- ração do $17 \beta$-Estradiol das amostras comerciais (gel e adesivo) e do gel simulado, empregando o método da adição de padrão de estradiol nas concentrações de 9,00, 12,00 e $15,00 \mathrm{mg} / \mathrm{mL}$, adotado pela AOAC, mostram uma recuperação de 98,90 a 99,04\%.

O método desenvolvido mostrou-se relativamente simples, preciso e exato, para determinação do $17 \beta$ Estradiol em formas farmacêuticas gel e adesivo transdérmico.

TABELA II - Resultado da repetitividade intradia das amostras de medicamento contendo $17 \beta$-estradiol, nas formas farmacêuticas gel e adesivo transdérmico

\begin{tabular}{lcccc}
\hline Amostra & Obtido & Média (\%) & $\begin{array}{c}\text { Desvio padrão } \\
\text { relativo }\end{array}$ & $\begin{array}{c}\text { Intervalo de } \\
\text { confiança da } \\
\text { média }(\mathbf{p}=\mathbf{0 , 0 5})\end{array}$ \\
\hline Simulada-gel $(0,75 \mathrm{mg} / 1,25 \mathrm{~g})$ & $0,75 \mathrm{mg} / 1,25 \mathrm{~g}$ gel & 100,0 & 1,43 & $0,75 \pm 0,01$ \\
Comercial-gel $(0,75 \mathrm{mg} / 1,25 \mathrm{~g})$ & $0,79 \mathrm{mg} / 1,25 \mathrm{~g}$ gel & 105,4 & 0,98 & $0,79 \pm 0,01$ \\
Comercial-adesivo $(3,20 \mathrm{mg} / \mathrm{adesivo})$ & $3,19 \mathrm{mg} /$ adesivo & 99,7 & 1,60 & $3,19 \pm 0,05$ \\
\hline
\end{tabular}

Os valores expressos como média, $n=10$ (gel) e $n=6$ (adesivo)

TABELA III - Resultados do teste de recuperação aplicado às amostras comerciais e simuladas de gel e adesivo transdérmico, em 3 níveis de concentração, em 3 dias

\begin{tabular}{lccc}
\hline Amostra & $\begin{array}{c}\text { Quantidade padrão } \\
\text { adicionado } \boldsymbol{\mu g} / \mathbf{m L}\end{array}$ & $\begin{array}{c}\text { Quantidade padrão } \\
\text { recuperado } \boldsymbol{\mu g} / \mathbf{m L} \\
*(\mathbf{D P})\end{array}$ & $\begin{array}{c}\text { Recuperação } \\
\mathbf{( \% )}\end{array}$ \\
\hline Comercial-gel $(12 \mu \mathrm{g} / \mathrm{mL})$ & 9,00 & $8,99(0,29)$ & 99,92 \\
& 12,00 & $11,95(0,23)$ & 99,58 \\
Simulado-gel $(12 \mu \mathrm{g} / \mathrm{mL})$ & 15,00 & $14,82(0,41)$ & 98,90 \\
& 9,00 & $8,94(0,17)$ & 99,31 \\
Comercial-adesivo $(12,80 \mu \mathrm{g} / \mathrm{mL})$ & 12,00 & $11,98(0,24)$ & 99,86 \\
& 15,00 & $14,86(0,29)$ & 99,04 \\
& 10,24 & $10,15(0,13)$ & 99,15 \\
& 12,80 & $12,74(0,31)$ & 99,53 \\
& 15,36 & $15,32(0,43)$ & 99,72 \\
\hline
\end{tabular}

TABELA IV - Dados do teste de linearidade do método colorimétrico de análise do 17ß-estradiol, em 3 dias diferentes, de forma farmacêutica gel

\begin{tabular}{lcccc}
\hline Concentração (mg/mL) & $\mathbf{\%}$ & $\mathbf{1}^{\circ}$ ensaio* A (DP) & $\mathbf{2}^{\circ}$ ensaio* A (DP) & $\mathbf{3}^{\circ}$ ensaio* A (DP) \\
\hline 12 & 50 & $0,288(0,003)$ & $0,311(0,001)$ & $0,310(0,005)$ \\
18 & 75 & $0,428(0,002)$ & $0,461(0,005)$ & $0,466(0,009)$ \\
24 & 100 & $0,582(0,002)$ & $0,611(0,009)$ & $0,634(0,003)$ \\
\hline
\end{tabular}

*os valores estão expressos como média, $\mathrm{n}=3$ e (desvio padrão) 


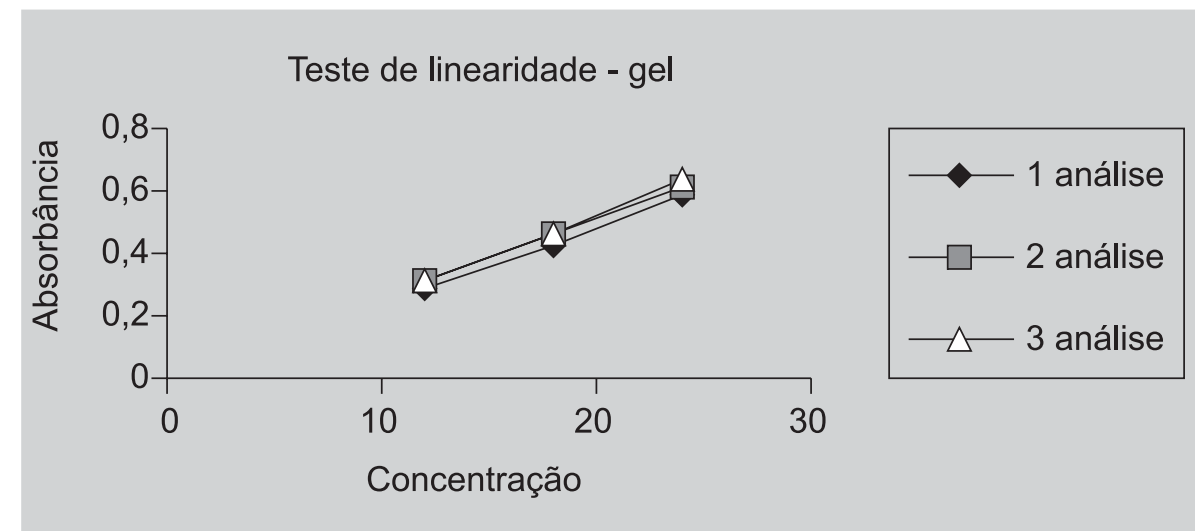

FIGURA 4 - Linearidade do método colorimétrico de análise do 17ß-estradiol, em forma farmacêutica gel, em 3 dias diferentes.

\section{ABSTRACT \\ Determination of $17 \beta$-estradiol in dosage formulations: gel and patch}

A visible spectrophotometry method was developed for quantification of $17 \beta$-estradiol in gel and in patch, tranformations by using diazotised sulfanilamide as the colorimetric reagent. The azo-compound formed showed a maximum absorption in $480 \mathrm{~nm}$, and the reaction was linear in a concentration range from 10,0 to $28,0 \mu \mathrm{g} / \mathrm{mL}$. When applied to dosage formulations it showed to be precise and accurate.

UNITERMS: 17 $\beta$-estradiol. Visible spectrophotometry. Gel. Patch. Azo-compound.

\section{REFERÊNCIAS BIBLIOGRÁFICAS}

\section{ASSOCIATION OF OFFICIAL ANALYTICAL}

CHEMISTS. Drugs. In: . Official methods of analysis of AOAC International. 17.ed. Gathersbury: AOAC International, 2000. part.IV.

DEF 2003/04: Dicionário de especialidades farmacêuticas. 32.ed. Rio de Janeiro: Publicações Científicas, 2003. $1294 \mathrm{p}$.

HUPPERT, L.C. Hormonal replacement therapy: benefits, risks, doses. Med. Clin. North Am., Orlando, v.71, n.1, p.23-39, 1987.
ICH-Harmonized Tripartite Guideline. Validation of Analytical Procedures: Methodology. Q2B. 1996 p.1-8. Disponível em: http://www.ich.org/MediaServer.jser?@_ID= 418@_MODE=GLB. Acesso em: 05 jan. 2004.

KOTIYAN, P.N.; VAVIA, P.R. Stability indicating HPTLC method for the estimation of estradiol.J. Pharm. Biomed. Anal., Amsterdam, v.22, p.667-671, 2000.

NELSON 2, H.D. Postmenopausal estrogen for treatment of hot flashes. JAMA, J. Am. Med. Assoc., Chicago, v.291, n.13, p.1621-1625, 2004.

SALCI, B.; BIRYOL, I. Voltametric investigation of $\beta$ estradiol.J. Pharm. Biomed. Anal., Amsterdam, v.28, n.3/ 4, p.753-759, 2002.

STEPHENSON, J. FDA orders estrogen safety warnings. Agency offers guidance for HRT use. JAMA, J. Am. Med. Assoc., Chicago, v.289, n.5, p.537-538, 2003.

TORAL, M.I.; SORO, C.; RICHTER, P. Simultaneous determination of estradiol and medroxyprogesterone acetate in pharmaceutical formulations by secondderivative spectrometry. J. AOAC Int., Gaithersburg, v.85, n.4, p.883-888, 2002.

UNITED States Pharmacopeia: USP26. The National Formulary: NF21. Rockville: United States Pharmacopeial Convention, 2003. p.742-744, 2054, 2176-2177, 2439-2442.

Recebido para publicação em 31 de maio de 2004. Aceito para publicação em 12 de novembro de 2004. 